Les années

70

\title{
LA PENSÉE COMMUNICATIONNELLE DANS LES ANNÉES SOIXANTE-DIX : CRITIQUE DES MÉDIAS ET ÉMERGENCE DE NOUVELLES PRATIQUES ALTERNATIVES
}

\author{
Serge Proulx
}

A la mémoire de Yves de la Haye mort en 1983

Je me sens particulièrement heureux d'être parmi vous aujourd'hui afin de rendre compte du développement de la pensée communicationnelle pendant la décennie soixante-dix. Comme j'ai été personnellement impliqué dans certains moments privilégiés de développement de notre champ d'étude pendant cette période, cette tâche de remémoration fut pour moi prenante et instructive. J'ai terminé en effet mes études de doctorat à Paris dans les premières années de la décennie, puis j'ai commencé à enseigner les communications à l'université dans cette période de forte contestation sociale au Québec.

1 Professeur titulaire, Département des communications, Université du Québec à Montréal. 
Pendant mes premières années comme professeur à l'université du Québec à Montréal (UQAM), j'ai participé activement à la création d'un département d'étude et de formation à la communication dans cette institution. Je me suis ainsi retrouvé à 29 ans, premier directeur d'un programme de baccalauréat spécialisé en communication à l'UQAM dans un contexte de société où la communication devenait progressivement un enjeu stratégique important.

C'était une époque où la fonction critique de l'université était pleinement reconnue. Pour le jeune professeur formé à la sociologie critique que j'étais, le projet de créer un nouveau lieu institutionnel universitaire en communication était perçu comme un défi exaltant parce qu'il supposait de pouvoir mobiliser un ensemble non négligeable de ressources intellectuelles et matérielles pour penser critiquement ce nouveau champ de problèmes en émergence. Et il s'agissait non seulement d'effectuer un travail de critique théorique de la communication mais aussi de mettre en place un lieu de formation pratique d'où sortiraient éventuellement de jeunes diplômés en communication susceptibles de développer des manières alternatives de pratiquer la communication dans une société québécoise en bouillonnement. Le défi à la fois théorique et pratique de ce projet pédagogique de formation à la communication pouvait ainsi se traduire par la double question suivante: comment susciter et transmettre des savoir-faire et des savoirs critiques concernant la communication? Comment ces nouvelles pratiques de communication pouvaient-elles participer aux mouvements sociaux de transformation qui agissaient la société toute entière?

\section{L'émergence de nouvelles pratiques alternatives en communication}

La décennie soixante-dix fut une période historique privilégiée du point de vue de l'émergence de nouvelles pratiques de communication. Au Québec, la décennie soixante avait été orientée vers l'implantation d'une série de réformes sociales initiées à l'occasion de ce que nous avons appelé la Révolution tranquille. Cette stratégie d'implantation supposait le recours à une panoplie de techniques d'intervention et d'animation. Après cette vague de l'animation sociale des années soixante, ce seront les techniques de communication qui seront mises de l'avant pour susciter la participation populaire au changement 
social : techniques d'animation de groupe bien sûr mais surtout, à partir de l'été 1968 , l'on voit apparaître une invention technique qui va susciter un fort enthousiasme à la fois chez les cinéastes concernés par l'engagement social et parmi divers militants des milieux populaires. Je veux parler ici du Porta-Pak de Sony, la première caméra vidéo portable et à prix modique qui devint rapidement la source de fantasmes utopiques pour divers militants culturels. Cet objet technique étant facile d'usage, il n'y avait donc pas besoin de formation spécialisée pour l'utiliser. Des membres d'organisations populaires ou communautaires se montrèrent très intéressés à produire leurs propres images et à intervenir socialement au moyen de la communication. On pourrait dire qu'il y eut, à l'échelle nordaméricaine, émergence et déploiement d'un quasi-mouvement social autour d'un ensemble impressionnant de pratiques alternatives en communication suscitées par l'usage de la vidéo portable.

L'émergence de ces nouvelles pratiques de communication coïncidait avec un système de convictions idéologiques qui s'ancrait solidement chez certains partisans du changement social, à savoir que c'était par le biais des médias que le changement pourrait advenir. Cette idéologie de la communication était bien sûr en résonance avec les thèses de Marshall McLuhan qui avaient été particulièrement médiatisées pendant la seconde moitié de la décennie soixante. Il s'agissait donc de briser le monopole de l'imposition culturelle détenu jusque-là par les grands réseaux de radiodiffusion en multipliant des pratiques sociales qui se définissaient comme des alternatives à cette manière dominante de concevoir l'usage des médias. Le Porta-Pak devenait le symbole et l'outil concret pour pratiquer une "guérilla culturelle" contre la communication de masse devenue le modèle à abattre. Il devenait possible d'imaginer et d'inventer de nouvelles manières d'utiliser la télévision. La vidéo permettait ainsi de démystifier les images télévisuelles dominantes : l'on pouvait, par exemple, produire des contre-publicités, détourner des images en leur juxtaposant des bandes sonores dissonantes, les monter différemment en alternant images de fiction et images documentaires, jouer avec les images fixes et les ralentis, etc. Il devenait aussi possible de déconstruire électroniquement les images, ce qui donna lieu à une série d'événements réalisés par des artistes adeptes de ce qu'ils appelaient le "vidéo feed-back".

Les lieux d'expérimentations sociales avec la vidéo se multipliaient: dans la rue, sur les lieux de luttes politiques ou syndicales, 
dans les musées qui accueillaient les premières installations vidéo, dans les écoles où les enfants étaient invités à réaliser leurs propres images, dans les groupes de thérapie collective où la vidéo pouvait servir de miroir auto-analytique, etc. Des militants politiques s'organisaient en "groupes d'intervention vidéo" prêts à intervenir sur les lieux de grèves ou de conflits ouvriers. D'autres artisans de la vidéo l'introduisaient dans les milieux du cinéma engagé : je pense ici notamment au programme "Challenge for Change" mis en place par des cinéastes de l'Office national du film (ONF) au Canada. D'autres encore introduisirent la vidéo dans les milieux organisationnels, soit comme instrument de changement, soit pour fins de production de documents institutionnels propres. Des réseaux de vidéastes se mirent en place, des associations de militants culturels plus ou moins engagées politiquement virent le jour, des vitrines apparurent (par exemple, "Le Vidéographe" à Montréal). Dans le secteur de l'éducation, forts du constat que la télévision de masse s'avérait un modèle inefficace de télévision éducative, des éducateurs cherchaient à définir de nouvelles stratégies éducatives en partant des besoins spécifiques de formation de groupes particuliers : le recours à la vidéo devenait un outil éducatif prometteur.

En combinant la vidéo portative aux possibilités de distribution des images par le moyen du câble, certains artisans imaginèrent l'un des concepts les plus spectaculaires de cette époque, et qui fut particulièrement développé au Québec : la télévision communautaire. Nous ne pouvons ici -dans le cadre restreint de cet article-faire une analyse approfondie de ce phénomène important. Disons simplement que ces pratiques de télévision communautaire furent des lieux d'expérimentations intéressants qui permirent de combler des besoins d'information et de communication dans un contexte de forte disparité régionale du point de vue de l'accès aux moyens de diffusion. Parallèlement, ces télévisions communautaires furent le théâtre d'un bouillonnement social local où se trouvaient à l'œuvre différents types d'acteurs sociaux parfois associés, parfois en conflits et qui cherchaient à faire valoir leurs intérêts : des acteurs gouvernementaux qui se livraient à des luttes politiques nationales par le biais de ces terrains d'intervention locale; des acteurs industriels et commerciaux qui étendaient leur publicité et leurs réseaux de services ; enfin, des artisans idéologiquement hétérogènes, plus ou moins conscientisés socialement, et se retrouvant parfois au cœur de luttes concurrentielles. 


\section{L'affirmation d'une pensée critique concernant la communication}

En Europe et en Amérique du nord, particulièrement dans les universités, la décennie soixante-dix est une période privilégiée pour l'affirmation et la cristallisation d'une pensée critique en communication. Les mouvements sociaux étudiants de la fin des années soixante ont sans doute agi comme un catalyseur de la conscience sociale et politique de plusieurs universitaires des pays occidentaux. On avait coutume jusque-là d'opposer la pensée philosophique et spéculative des intellectuels européens à la démarche empiriste des chercheurs américains en sciences sociales appliquées, les premiers étant $a$ priori plus proches d'une attitude critique, les seconds s'identifiant davantage au paradigme positiviste. Avec les années soixante-dix, cette distinction s'efface graduellement: certains chercheurs européens s'ouvrent aux études empiriques pendant que des chercheurs américains choisissent d'approfondir les fondements théoriques de leur démarche de terrain. Une nouvelle distinction se substitue alors à la première. Il y a, d'une part, des spécialistes en sciences sociales qui ne semblent chercher qu'à accumuler de nouvelles données empiriques sans préoccupation apparente pour la production d'hypothèses ou de propositions théoriques qui arrimeraient la compréhension des phénomènes de communication à des contextes plus larges. D'autre part, une nouvelle communauté de chercheurs critiques voit le jour, qu'ils soient européens ou américains : ils posent d'emblée comme fondamental pour la compréhension de la communication sociale le lien qui unit le fonctionnement des médias aux rapports sociaux de pouvoir.

Dans le secteur des communications, cette pensée critique se déploie dans plusieurs directions. Le découpage classique du mode de production en trois instances (idéologique, économique, politique) proposé à l'époque par les marxistes permet déjà de cerner trois sphères importantes de la pensée critique en communication, à savoir : l'action idéologique des médias, le développement contradictoire des industries culturelles, et enfin, les transformations d'un espace public de discussion politique qui est au fondement même du fonctionnement de la démocratie. L'on pourrait faire référence également à deux autres dimensions clés de ces travaux critiques : d'une part, les recherches sur l'internationalisation des systèmes de communication et d'autre part, les réflexions sur le rôle politique des intellec- 
tuels face aux médias. J'effectuerai un rapide survol de ces cinq dimensions de la pensée critique en communication.

\subsection{L'action idéologique des médias}

Dans le prolongement des travaux de la sémiologie des communications de masse -en résonance notamment avec la démarche de déconstruction des mythologies quotidiennes effectuée par Roland Barthes- plusieurs chercheurs critiques orientent leur travail vers l'analyse de l'action idéologique des médias. Marqués par la conception althussérienne de l'idéologie, ils définissent les médias en tant qu'appareil idéologique d'État et cherchent une mise à plat des mécanismes par lesquels la société se reproduit à l'avantage des classes dominantes. Les analyses de ces chercheurs - qui postulent $a$ priori que les médias reproduisent l'idéologie dominante- manquent souvent de subtilité tellement elles sont embuées de la «langue de bois» imposée par la vulgate structuralo-marxiste. Elles sont essentiellement orientées vers l'étude des contenus médiatiques et supposent par conséquent, qu'une déconstruction des contenus des messages est un travail nécessaire et suffisant pour mettre à jour l'action idéologique des médias. Le constat de cette insuffisance méthodologique et épistémologique constituera le point de départ des réflexions de Stuart Hall qui proposera vers 1975 un nouveau programme de recherche sur le travail idéologique des médias pour les Cultural Studies britanniques orienté vers une prise en compte des actions de décodage effectuées au point de réception. Cette nouvelle orientation dans le travail des chercheurs critiques en communication constituera un véritable tournant dans l'étude des effets idéologiques des médias. Les chercheurs seront obligés d'admettre, comme le signalera plus tard Daniel Dayan, que ce n'est pas le message émis ou diffusé qui peut enclencher l'effet idéologique mais bien le message reçu. Ce nouveau type de recherches sur la réception médiatique connaîtra son apogée durant la décennie quatre-vingt.

\subsection{Le développement contradictoire des industries culturelles}

S'inscrivant dans la continuité des travaux de Horkheimer et Adorno, de l'école de Francfort, d'autres chercheurs d'orientation 
critique définissent le système des médias en tant que système de production industrielle de la culture. Ils proposent une critique de la modernité à partir de la notion d'industrie culturelle. Ils analysent le fonctionnement des industries culturelles dans le contexte du développement parallèle de la logique de marché et de la mise en place d'un système de régulation étatique visant à encadrer l'expansion des médias et des technologies de communication. Ces analyses ont mis en relief la tendance à la marchandisation apparemment inscrite dans le développement des systèmes de communication des sociétés occidentales depuis trente ans, tendance qui, dans beaucoup de pays, semble fragiliser le fonctionnement du système des médias en tant que service public.

\subsection{Les transformations des espaces publics}

Les débats autour de la fonction de service public des médias vont pouvoir s'approfondir et se complexifier à l'aide du concept d'espace public défini par le philosophe Jurgen Habermas dans un ouvrage publié en allemand en 1962 et finalement traduit en langue française en 1978. En étudiant le passage entre le modèle d'espace public issu des Lumières vers un modèle d'espace public propre à la société bourgeoise du XIX ${ }^{\mathrm{e}}$ siècle, Habermas incite les chercheurs contemporains à analyser les transformations de cet espace rationnel de discussion publique - essentiel au fonctionnement démocratiqueprovoquées par la présence des médias modernes de diffusion.

\subsection{L'internationalisation des systèmes de communication}

D'autres chercheurs ont mis en relief l'internationalisation des systèmes de communication de même que les effets de ce mouvement de concentration économique sur une homogénéisation possible au niveau planétaire d'une culture populaire médiatique, en particulier sous l'influence des industries du divertissement des États-Unis d'Amérique. En outre, la mise sur pied par l'Unesco, en décembre 1977, de la Commission McBride proposant l'instauration d'un Nouvel Ordre mondial de la communication et de l'information, a catalysé la réflexion critique autour de l'enjeu d'un conditionnement culturel des pays du tiers monde par un système mondial des médias 
contrôlé essentiellement par les États-Unis et le Japon. À l'idéologie de la libre circulation de l'information portée par les grands trusts de la communication internationale, la pensée critique opposait la thèse d'une unidirectionnalité dans la circulation des biens informationnels à l'échelle planétaire.

\subsection{La position politique des intellectuels face aux médias}

La publication en 1970 par le penseur critique allemand Hans Magnus Enzensberger d'un article intitulé «Constituents of a theory of the media» dans la revue marxiste américaine New Left Review, suscita un débat fort intéressant au sein de la gauche américaine à propos de la position des intellectuels face aux médias. Enzensberger secouait l'indifférence affichée jusque-là par les penseurs de gauche à l'égard des médias. Dans un premier temps, Enzensberger insistait sur l'importance croissante des médias en tant que forces spécifiques de production dans le nouveau secteur des industries de la conscience. Il constate ensuite le hiatus entre la position des tenants des contrecultures qui expérimentent dans l'enthousiasme avec les nouvelles technologies pour imaginer des utilisations alternatives des médias -il s'agit des militants culturels dont j'ai parlé plus tôt- alors que les penseurs de la gauche socialiste marginalisent le rôle des médias et s'en tiennent à une analyse traditionnelle des forces de production en présence.

Selon ce philosophe critique, la gauche semble sortir perdante de cette situation où d'un côté, les contre-culturels acceptant les nouveaux médias semblent par ailleurs incapables de formuler une analyse politique solide des pratiques nouvelles et où de l'autre côté, les activistes socialistes refusent de voir et donc d'analyser les possibilités révolutionnaires nouvelles suscitées par les nouveaux médias. La position des intellectuels de gauche semble se restreindre à une analyse de la propriété capitaliste des médias alors que l'essentiel est ailleurs, dans les possibilités que représentent les médias, soit pour manipuler la formation de l'opinion publique, soit pour démocratiser l'espace public politique. Cette réflexion débouchera sur un nouveau type de questionnement autour du double rôle possible des médias, soit répressif (aliénant), soit émancipateur (libérateur). Cette problématique construite autour du couple «aliénation - émancipation» 
structurera pendant les années 1970 et 1980 , un bon nombre de travaux portant sur les usages possibles des médias.

Dans son « Requiem pour les media » (1972), Jean Baudrillard rebondit sur la réflexion de Enzensberger en défendant la thèse que la stratégie révolutionnaire en matière de médias est celle de la transgression des codes :

La pensée d'Enzensberger, elle, se veut optimiste et offensive. Les media sont actuellement sous le monopole des classes dominantes, qui les détournent à leur profit. Mais leur structure, elle, reste "fondamentalement égalitaire", et c'est à la pratique révolutionnaire de dégager cette virtualité inscrite en eux, mais pervertie par l'ordre capitaliste (...).

Les exemples [qu'Enzensberger] donne sont intéressants en ceci qu'ils dépassent une "dialectique" de l'émetteur et du récepteur. On y retrouve en effet un procès de communication immédiate, non filtrée par des modèles bureaucratiques, une forme d'échange originale parce qu'en fait il n'y a plus d'émetteurs ni de récepteurs, mais des gens qui se répondent. Le problème de la spontanéité et de l'organisation n'est pas ici dépassé dialectiquement, il est transgressé dans ses termes'.

Ce type de débats va donc participer d'un mouvement de légitimation d'un discours critique concernant les médias et leur rôle décisif (mais subtil) dans l'imposition de l'idéologie dominante. Aux dires de Baudrillard, les médias agiraient plus efficacement à travers la forme qu'ils imposent aux rapports d'échange entre les individus (non-réciprocité) que par les contenus des messages diffusés. Baudrillard rejoint ici, par la gauche, l'une des thèses de McLuhan.

\section{La communication devient un enjeu de société}

C'est dans les décennies soixante et soixante-dix que la communication devient véritablement un enjeu de société. Je vais illustrer mon propos à partir de l'histoire du Québec. Dans le prolongement de la Révolution tranquille des années soixante -appellation que j'ai utilisé précédemment et qui recouvre un vaste mouvement de transformations sociales initiées par l'État québécois s'introduisant dans les secteurs de l'éducation, de la santé et de la sécurité sociale de

1 J. BAUDRILlard, Pour une critique de l'économie politique du signe, Paris, Gallimard, 1972, p. 205 et 226. 
même que dans la définition des orientations sociales du développement socioéconomique- apparaît un mouvement d'institutionnalisation d'un ensemble de pratiques professionnelles spécifiques en communication contribuant ainsi progressivement à définir la communication comme enjeu social. La communication devient en effet un sujet de réflexions publiques et une instance de médiation qu'il vaut mieux contrôler parce que l'on comprend que le contrôle de la communication est une façon de s'assurer d'un pouvoir sur l'organisation, qu'il s'agisse de l'organisation que constitue chaque entreprise privée ou plus globalement, de l'organisation politique dans son ensemble. Les technocrates aux commandes des grandes entreprises et de l'appareil gouvernemental vont donc participer activement à ce mouvement d'institutionnalisation des pratiques de communication.

Déjà l'implantation en 1952 des deux premières chaînes de télévision de Radio-Canada, en contribuant à transformer significativement les loisirs et la vie quotidienne des citoyens et citoyennes, avait entraîné une première prise de conscience des «impacts sociaux» des moyens modernes de diffusion (cinéma, presse, radio, télévision) et de la «menace» qu'ils représentaient pour la vie familiale et les valeurs catholiques. Cette conscientisation était présente dans les premières réflexions d'organismes religieux comme le Centre catholique du cinéma, de la radio et de la télévision (qui deviendra plus tard l'Office des communications sociales) ou de groupes civiques de pression comme l'Institut canadien d'éducation des adultes (ICEA).

Ce mouvement de conscientisation autour de la communication comme enjeu social s'institutionnalise rapidement au tournant des décennies soixante / soixante-dix en raison des nombreuses interventions de l'État (mise sur pied du CRTC, organisme de règlementation canadien ayant pour mandat d'accorder les permis de diffusion et ayant, par conséquent, droit de regard sur les programmations ; création des ministères canadien et québécois des communications en 1968 et 1969 ; établissement de Radio-Québec en 1972). On assiste d'ailleurs, à cette époque, à l'éclatement d'une guerre de juridictions entre les gouvernements québécois et canadien autour des enjeux que constituaient le contrôle de la radiodiffusion et celui de la câblodistribution. Cette guerre sera finalement remportée par le gouvernement canadien. Cette volonté de contrôle gouvernemental du secteur des communications va d'ailleurs se poursuivre bien au-delà des années 1970 , à travers les efforts que les deux ordres de gouvernements vont 
déployer pour s'impliquer dans le développement des nouvelles technologies d'information et de communication, ce qui les conduira à la mise sur pied de nombreux groupes d'étude et à la publication de nombreux énoncés de politiques concernant le développement économique devant effectuer un virage technologique, l'avènement de la société d'information et plus récemment, la mise en place des autoroutes de l'information. Depuis les années soixante-dix, l'État aura tendance à épouser de plus en plus les intérêts économiques et industriels des entreprises privées et à progressivement délaisser les politiques ayant une finalité avouée de développement social ou culturel.

Mais revenons à la décennie 1970 : parallèlement aux actions des gouvernements, des syndicats se constituent regroupant les spécialistes en communication (Fédération nationale des communications, Fédération professionnelle des journalistes du Québec) ; des départements universitaires en communication sont créés (Concordia, Laval, Montréal, UQAM) assurant une formation académique aux futurs professionnels et développant une expertise en recherche et évaluation des impacts sociaux ; des firmes privées de recherche dans le secteur des médias et des communications sont mis sur pied et contribuent à dresser un portrait des publics, de leurs habitudes de consommation et d'usages des médias; les médias eux-mêmes diffusent un discours social à propos de leur propre influence (dans le prolongement de l'effet McLuhan). Tout cela contribue à cerner approximativement un nouveau champ de pratiques professionnelles qui aura tendance à se développer significativement au cours des décennies qui vont suivre.

L'importance prise par les spécialistes de communication dans des domaines aussi variés que le fonctionnement des organismes publics et parapublics, le management d'entreprises privées relevant de multiples secteurs, la gestion de la communication politique (niveaux local, national, international), la production massive des marchandises culturelles - la liste pourrait s'allonger largement- se poursuivra sans interruption jusqu'à nos jours. Le champ professionnel de la communication a pris avec le temps une importance extrêmement grande. Certains analystes sociaux se montrent d'ailleurs parfois scandalisés quand les budgets de certains services de communication (de gouvernements, d'entreprises, de syndicats) sont rendus publics. 


\section{Conclusions}

La fin des années soixante-dix fut marquée à la fois par la mort lente des fantasmes utopiques associés aux nouveaux médias de l'époque, et par le début d'un mouvement subtil de régression de la pensée critique. Dans le contexte du triomphe du libéralisme pendant les deux dernières décennies, d'une logique de plus en plus dominante de marchandisation des biens communicationnels et d'imposition des intérêts des entreprises privées sur les gouvernements, une idéologie professionnaliste de la communication s'est progressivement substituée aux velléités critiques et utopiques de la décennie précédente. Je pense qu'il devient urgent aujourd'hui, pendant qu'il en est encore temps, de renouer avec l'héritage de cette pensée critique née dans la décennie soixante-dix.

Nous sommes aujourd'hui dans une situation où certaines tendances du développement des médias sont exacerbées. Je pense, entre autres, à la marchandisation à outrance des échanges et au triomphe de la logique de marché, à l'individualisation des pratiques de consommation, aux formes de spectacularisation de plus en plus vive des produits communicationnels, à l'enchevêtrement des industries du divertissement, de la publicité et de l'information, à la concentration économique et au contrôle par quelques grands trusts du mouvement de mondialisation des médias, et finalement, à la privatisation croissante de la propriété des médias encouragée par une généralisation des pratiques de dérégulation gouvernementale dans la plupart des sociétés occidentales. Or, la plupart de ces tendances alors en émergence avaient été mises en relief par la pensée critique de la décennie soixante-dix. Après le mouvement de régression de la pensée critique des derniers quinze ans, il me semble qu'il y a urgence pour qu'un plus grand nombre de chercheurs reviennent vers des problématiques critiques en approfondissant certaines des pistes ouvertes pendant la décennie soixante-dix.

Certains objets de recherche m'apparaissent à privilégier aujourd'hui, notamment la question de la construction des identités culturelles et celle de la constitution d'espaces publics qui feraient place à la parole des citoyens dans le présent contexte de mondialisation des médias. Il m'apparaît nécessaire d'insister sur le développement de perspectives interdisciplinaires, de stratégies méthodologiques à volets multiples et sur les synergies rendues possibles par la confrontation créatrice entre traditions de recherches jusqu'ici 
développées séparément -je pense, par exemple, à un projet sur lequel je travaille personnellement avec mon collègue Marc Raboy, où nous tentons de relier la tradition des travaux sur la réception à la tradition d'analyse des politiques publiques. À travers ce projet, c'est une symbiose entre l'économie politique de la communication et l'étude des pratiques culturelles de communication qui est visée.

De la même manière, il m'apparaît pertinent de confronter -pour éventuellement faire converger- les traditions de recherches concernant les médias traditionnels aux perspectives développées autour des nouvelles technologies d'information et de communication. Finalement, la prise en compte de la problématique de la réflexivité du travail du chercheur est essentielle. Il m'apparaît capital, en effet, de prendre conscience par exemple, que le choix de nos dispositifs de collecte de données va déteindre en profondeur sur le processus de construction et sur la nature même de ces données. De manière similaire, le récit que construira l'observateur à partir des données qu'il aura recueilli sera marqué par ses postulats implicites et par ses présupposés épistémologiques. La prise en compte de la question de la réflexivité suppose un travail nécessaire d'explicitation de ces postulats implicites. Voilà peut-être où se situe le travail critique aujourd'hui.

\section{Bibliographie}

J. BAUDRILLARD, Pour une critique de l'économie politique du signe, Paris, Gallimard, 1972.

P. BRETON \& S. PROULX, L'explosion de la communication, nouvelle édition, Paris, La Découverte/Poche, 1996.

H.M. ENZENSBERGER, "Constituents of a Theory of the Media", New Left Review, $\mathrm{n}^{\circ}$ 64, 1970, New York, p. 13-36.

S. PROULX, "Définitions de la communication: réponses à un questionnaire", MEI "Médias et information", n²4, 1996, Université de Paris VIII, p. 33-37.

S. PROULX \& P. VALIÈreS, (éds.), Changer de société. Déclin du nationalisme, crise culturelle, alternatives sociales au Québec, Montréal, Québec/Amérique, 1982.

M. SHAMBERG, Guerrilla Television, New York, Holt Paperback, 1971. 